Check for updates

Cite this: RSC Adv., 2019, 9, 7440

Received 13th December 2018

Accepted 27th February 2019

DOI: $10.1039 / c 8 r a 10222 h$

rsc.li/rsc-advances

\section{A sandwich-type catalytic composite reassembled with a birnessite layer and metalloporphyrin as a water oxidation catalyst $\dagger$}

\author{
Fan Liu, Liming Wang, Weijun Yang, (D) * Enqing Liu and Can Huang
}

High purity birnessite was synthesized and exfoliated into a negatively charged monolayer structure. A positively charged 5, 10, 15, 20-tetrakis (4-aminophenyl) manganese porphyrin (MnTAPP) was synthesized. Driven by the electrostatic force and the coordination effect of the amino nitrogen on the manganese ion in birnessite, the single-layer birnessite was reassembled with MnTAPP, forming a new sandwich-type catalytic composite MnTAPP@bir. The structure and chemical properties of the composite were characterized by X-ray diffraction (XRD), Fourier transform infrared spectroscopy (FTIR), scanning electron microscopy (SEM), transmission electron microscopy (TEM), thermogravimetric analysis (TGA) and Brunauer-Emmett analysis (BET). Electrocatalytic studies showed that the MnTAPP@abir exhibited an overpotential for water oxidation of $450 \mathrm{mV}$ (at $10 \mathrm{~mA} \mathrm{~cm}{ }^{-2}$ ) and a Tafel slope of $121.5 \mathrm{mV} \mathrm{dec}{ }^{-1}$ compared to birnessite with $700 \mathrm{mV}$ (at $10 \mathrm{~mA} \mathrm{~cm}^{-2}$ ) and $230 \mathrm{mV} \mathrm{dec}{ }^{-1}$. Impedance spectroscopy results suggested that the charge transfer resistivity of MnTAPP@bir was significantly lower than that of birnessite, suggesting that MnTAPP in the interlayer increased the conductivity of birnessite. Through a chronoamperometry test, the new material also showed excellent stability within $4000 \mathrm{~s}$.

\section{Introduction}

With the development of society and economy, Energy consumption is rising and traditional energy sources such as coal, oil and natural gas are drying up. As the price of fossil fuels fluctuates and global climate changes rapidly, researchers endeavor to find clean, efficient and renewable energy. ${ }^{1}$ Among numerous hydrogen production technologies, water splitting with solar energy has been highlighted. This process requires a lot of electrons and protons as reducing agents. ${ }^{2-4}$ The water oxidation process can provide sufficient electrons and protons for this process. Thus, water oxidation is the critical step for hydrogen production technology. However, there are thermodynamic disadvantages in the water oxidation process, requiring the external conditions to provide higher energy. ${ }^{5}$ Therefore, in order to promote the water oxidation process, it is necessary to develop an efficient, stable, and inexpensive catalyst. However, Pt, $\mathrm{RuO}_{2}$, and $\mathrm{IrO}_{2}$, which are now wellperforming oxygen evolution reaction (OER) catalysts, are expensive, and their natural reserves are small, limiting their practical application. ${ }^{6}$

Birnessite is a layered inorganic material with cations between layers. Its lamellar structure is formed by edge-sharing

College of Chemistry and Chemical Engineering, Hunan University, Changsha 410082, China.E-mail: wjyang@hnu.edu.cn

$\dagger$ Electronic supplementary information (ESI) available. See DOI: 10.1039/c8ra10222h manganese octahedra $\left(\mathrm{MnO}_{6}\right) \cdot{ }^{7}$ In terms of chemical theory, the valence of manganese in birnessite should exist at +4 . But in actuality of birnessite, due to the presence of interlaminar structure defects, some of the $\mathrm{Mn}^{4+}$ is replaced by $\mathrm{Mn}^{3+}$, resulting in the entire sheet being negatively charged. In order to maintain electrical neutrality across the entire layer, $\mathrm{Na}^{+}$or other cations are present in the interlayer structure of birnessite. In addition, there will also be some water molecules present in the layers, which have the effect of stabilizing the birnessite layered structure. ${ }^{8}$ Birnessite has the function of catalyzing water oxidation, its low price and environmental protection, so it attracts much attention from researchers. ${ }^{\mathbf{9 - 1 1}}$ However, low conductivity and poor cycle performance limit its application in the field of water oxidation. ${ }^{\mathbf{1 2 , 1 3}}$ Metalloporphyrin compounds have a planar macrocyclic structure, excellent optical and electrical properties, and light and heat stability, making it an important application prospect in many fields, especially in the field of biomimetic catalysis. ${ }^{\mathbf{1 4 - 1 8}}$

Considering that the intercalations of metals and other substances into birnessite will enhance OER performance, and the success of porphyrin intercalates into layered materials such as insulating hexagonal boron nitride (h-BN) monolayer and thiosalicylic acid-modified $\mathrm{Mg}$-Al layered double hydroxides, ${ }^{\mathbf{1 9 - 2 6}}$ we hypothesized that intercalation of metalloporphyrin into layered birnessite can make use of the good stability of metalloporphyrin and the good electronic mobility in the porphyrin ring, thus strengthening the charge transfer performance of birnessite. In this paper, a new organic- 
inorganic composite, sandwich-type catalytic material MnTAPP@bir, was synthesized. Under the synergy of them, a water oxidation catalyst with higher electrocatalytic activity and better stability was obtained.

\section{Experimental}

\subsection{Materials}

All of the chemical compounds were purchased from Aladdin Reagent Co. Ltd, China, and were all analytical grades without special treatment unless otherwise mentioned.

\subsection{Preparation of MnTAPP@bir}

It can be found in the $\mathrm{ESI} \dagger$ that preparation method of 5,10,15,20-tetrakis(4-aminophenyl)manganese porphyrin (MnTAPP) and high purity birnessite.

In this study, high-purity birnessite was first placed in an acidic solution for $\mathrm{H}^{+}$exchange to synthesize birnessite with $\mathrm{H}^{+}$ between layers, and then tetramethylammonium hydroxide was added to exchange tetramethylammonium ions with $\mathrm{H}^{+}$, resulting in the expansion of the layer spacing, and then washed with deionized water to obtain an exfoliated monolayer of birnessite flakes. The positively charged MnTAPP was synthesized by the aforementioned method, and the laminates were selfassembled into the birnessite of the MnTAPP sandwich by the electrostatic attractive force of positive and negative charges and the affinity of the amino nitrogen to the manganese ions on the layer. MnTAPP@bir was synthesized on the basis of previous reports with slight modifications. ${ }^{23}$ The typical experimental steps are as follows:

First, $2 \mathrm{~g}$ of high-purity birnessite was added to $300 \mathrm{~mL}$ of $1 \mathrm{~mol} \mathrm{~L}^{-1} \mathrm{HNO}_{3}$ solution and stirred for $12 \mathrm{~h}$ at room temperature. Then washed with water, and dried at $70^{\circ} \mathrm{C}$. The resulting material is designated as $\mathrm{H}$-bir. $1 \mathrm{~g}$ of $\mathrm{H}$-bir was dispersed in $120 \mathrm{~mL}$ of deionized water. Then $12 \mathrm{~mL}$ of $25 \%$ tetramethylammonium hydroxide was added. The mixture was stirred at room temperature for $7 \mathrm{~d}$, and then the mixture was centrifuged at $12000 \mathrm{rpm}$ for $30 \mathrm{~min}$. The solid was washed three times with $200 \mathrm{~mL}$ deionized water and dried at $70{ }^{\circ} \mathrm{C}$ under vacuum to get exfoliated birnessite.

$0.0679 \mathrm{~g}$ MnTAPP and $0.4 \mathrm{~g}$ exfoliated birnessite were added into $100 \mathrm{~mL}$ deionized water and stirred for $24 \mathrm{~h}$ at $40{ }^{\circ} \mathrm{C}$. The reaction mixture was filtered and then washed with excess water and acetone, and finally dried for $12 \mathrm{~h}$ at $50{ }^{\circ} \mathrm{C}$. The resulting product is designated as MnTAPP@bir $(0.37 \mathrm{~g})$.

\section{Results and discussion}

\subsection{XRD analysis}

The powder XRD patterns of birnessite, exfoliated birnessite, acidified birnessite (H-bir) and MnTAPP@bir are shown in Fig. 1. It can be seen from the above figure that birnessite and $\mathrm{H}$-bir have obvious diffraction peak spectra at $12.3^{\circ}$ and $24.7^{\circ}$, which indicates that both of them have a layered structure. ${ }^{27}$ Therefore, it can be seen that birnessite still shows lamellar characteristic peaks after acidification of $\mathrm{HNO}_{3}$. It shows that in

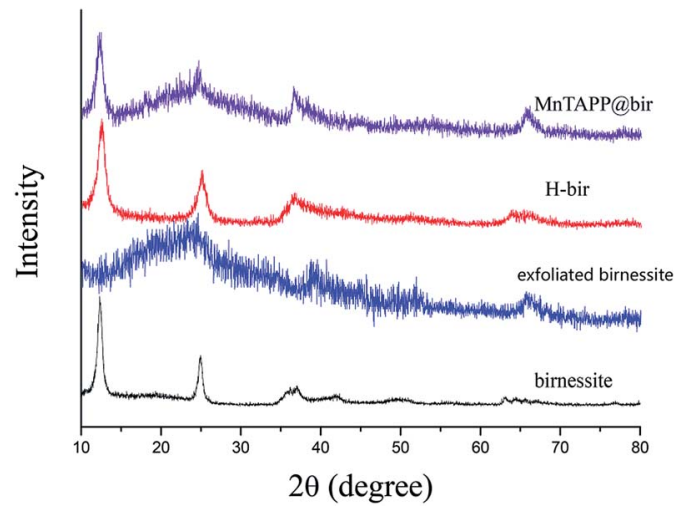

Fig. 1 XRD patterns of birnessite, exfoliated birnessite, $\mathrm{H}$-bir and MnTAPPabir.

the process of acidification, only the exchange of cations was performed, and the layered structure of birnessite was not destroyed. H-bir appeared more jagged peaks, which indicates that its crystallinity decreased and stability reduced after acidification.

After exfoliation, the characteristic peak of the birnessite disappears, and the layered structure has been destroyed. There are only some irregular jagged peaks. ${ }^{28}$ These peak patterns are caused by single-layered $\mathrm{MnO}_{2}$ nanoflakes after exfoliation. The specific reason of exfoliation is that during the process of exfoliation, tetramethylammonium cations enter the birnessite interlamellar structure through electrostatic force, thereby causing the expansion of the layered structure and thus causing the reduction of the interlayer force. The layered structure was broken by water washing to obtain a disordered exfoliated sheet.

The exfoliated birnessite has re-formed a layered structure through the combination of MnTAPP. This is because the surface of the sheet structure of $\mathrm{MnO}_{2}$ after exfoliation has a negative charge, while MnTAPP has positive charge. ${ }^{29}$ After they are mixed, the reassembly was achieved by electrostatic force, and the coordination effect of the amino nitrogen on the manganese ions in the layer also promotes the automatic assembly process. After reassembly, the crystallinity of $\delta-\mathrm{MnO}_{2}$ decreased.

\subsection{FTIR analysis}

Fig. 2a shows the infrared spectra of H-bir and birnessite. Both of them have absorption peaks near $1630 \mathrm{~cm}^{-1}$, which is caused by the bending vibration of interlayer water of birnessite. ${ }^{30}$ Acidified birnessite absorption peak is stronger. This is due to the fact that after the acidification, the $\mathrm{Na}^{+}$in the layers disappeared and more $\mathrm{H}_{2} \mathrm{O}$ between the layers was obtained. For both of them, there are absorption peaks in the range of 420$520 \mathrm{~cm}^{-1}$. This is due to the stretching vibration of $\mathrm{Mn}-\mathrm{O}$ bonds. ${ }^{31,32}$ The results of XRD analysis revealed that only the ion exchange process takes place in the acidification process. However, some changes have taken place inside the birnessite after acidification, resulting in the dissociation energy of $\mathrm{Mn}-\mathrm{O}$ 

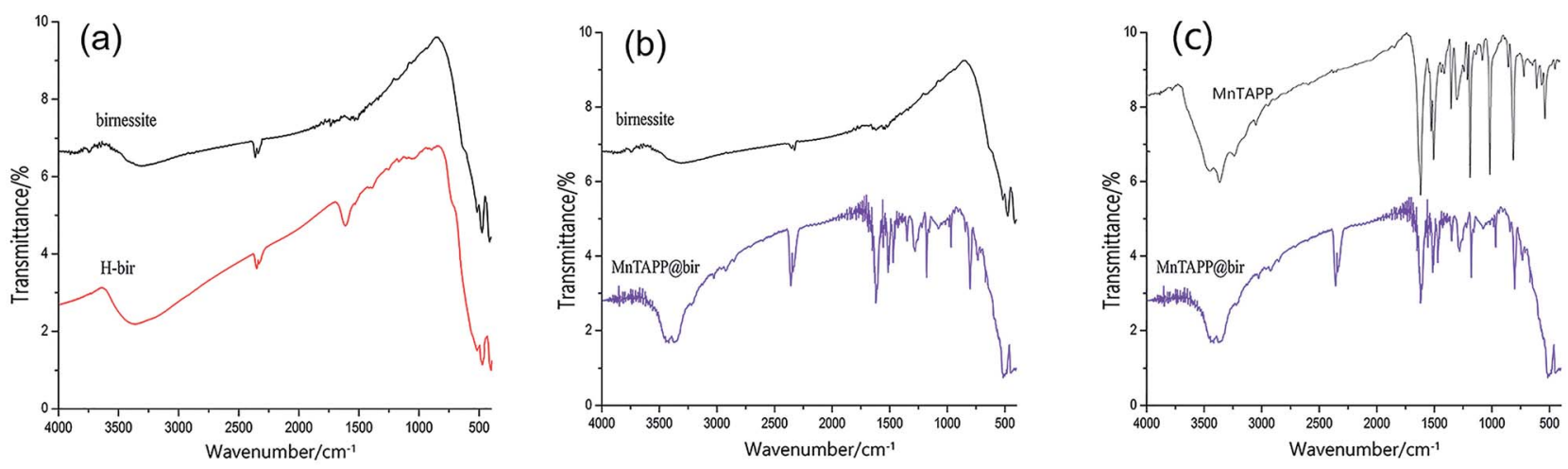

Fig. 2 FTIR spectrum of different products. (a) H-bir and birnessite; (b) birnessite and MnTAPP@bir; (c) MnTAPP and MnTAPP@bir.

bond being affected. Therefore, the XRD characteristic diffraction peak of $\mathrm{Mn}-\mathrm{O}$ bond shifts.

Fig. 2b shows the infrared spectra of birnessite and MnTAPP@bir. Both of them show absorption peaks near $1630 \mathrm{~cm}^{-1}$. It can be seen that after loading, the MnTAPP@bir has a large amount of $\mathrm{H}_{2} \mathrm{O}$ between layers. Both have absorption peaks in the range of $420-520 \mathrm{~cm}^{-1}$, which is due to the stretching vibration of the $\mathrm{Mn}-\mathrm{O}$ bond. The re-assembled birnessite has absorption peaks at $1614 \mathrm{~cm}^{-1}, 1509 \mathrm{~cm}^{-1}$, due to the skeletal vibration of the benzene ring. ${ }^{33}$ The absorption peaks at $3380 \mathrm{~cm}^{-1}$ and $3450 \mathrm{~cm}^{-1}$ are the stretching vibration of $-\mathrm{NH}_{2}$. The absorption peaks at $1345 \mathrm{~cm}^{-1}$ represents the stretching vibration of the $\mathrm{C}-\mathrm{N}$ bond on the porphyrin ring. ${ }^{34,35}$ A characteristic peak appeared at $1008 \mathrm{~cm}^{-1}$, which is the characteristic absorption peak of the stretching vibration of $\mathrm{Mn}-\mathrm{N}$.

Fig. 2c shows the FTIR spectra of MnTAPP and MnTAPP@bir. The FTIR spectrum of MnTAPP@bir is essentially identical to that of MnTAPP itself, apart from the additional band at $500 \mathrm{~cm}^{-1}$ corresponding to the characteristic $\mathrm{Mn}-\mathrm{O}$ stretching vibration of birnessite. This confirms that the structure of MnTAPP has not been affected by intercalation into the interlayer galleries of birnessite.

\subsection{SEM and TEM characterization}

Fig. 3a and $\mathrm{b}$ are SEM photographs of birnessite. It can be seen that birnessite is a three-dimensional nano flower arrangement structure, and the petals are very smooth and uniform in thickness. Fig. 3c and d are SEM photographs of MnTAPP@bir. It can be seen that the reassembled product is still a threedimensional nanoflower-like structure, but the petals are not so smooth and uniform in thickness compared with the birnessite. The microstructure and morphology of the asassembled sheets were further observed using TEM.

As shown in Fig. 3e and f are TEM photographs of exfoliated birnessite. It can be seen that the birnessite has been exfoliated into a uniform thickness of nanoflakes, about $300-400 \mathrm{~nm}$ in size, less than $1 \mathrm{~nm}$ in thickness, and basically a single layer of flakes. Fig. $3 \mathrm{~g}$ and $\mathrm{h}$ are photos of MnTAPP@bir. It can be seen that there are obvious black stripes and patches on the lightcolored flakes. This is the image of MnTAPP inserted into the birnessite. The TEM images of MnTAPP@bir vary in depth due to multiple layers stacked. This result is consistent with the SEM and infrared characterization results, and further proves that by the action of electrostatic force and coordination effect, birnessite and MnTAPP reassembled to form a new stable sandwich nanoflower-like structure.

\subsection{TG analysis}

Fig. 4 is a TGA curve of weightlessness of MnTAPP@bir and birnessite in an Ar gas atmosphere, respectively. There are two stages in the pyrolysis of birnessite: the weight loss from room temperature to $120^{\circ} \mathrm{C}$ is mainly attributed to evaporation of the interlaminar water in the layered structure and water on the surface. The weight curve (TG) decreases further when the temperature is increased from $150{ }^{\circ} \mathrm{C}$ to $350{ }^{\circ} \mathrm{C}$, caused by further loss of interlayer moisture in the birnessite..$^{36}$ The decrease in the total amount during the temperature increase from $500{ }^{\circ} \mathrm{C}$ to $600{ }^{\circ} \mathrm{C}$ is caused by the loss of the oxygen molecules in the birnessite layer converting to the $\mathrm{Mn}_{2} \mathrm{O}_{3} \cdot{ }^{37} \mathrm{In}$ the curve of MnTAPP@bir, it can be seen that there is also the same weight loss of interlayer water or surface-adsorbed water from room temperature to $120{ }^{\circ} \mathrm{C}$. The difference is that after $400{ }^{\circ} \mathrm{C}$, the birnessite loaded with MnTAPP has significant weight loss, which is mainly due to the degradation of MnTAPP intercalated into the birnessite layer. On the other hand, reassembled birnessite may have more interlayer water, thus caused more weight loss compared with the birnessite.

\subsection{BET analysis}

Fig. $5 \mathrm{a}$ and $\mathrm{b}$ are the specific surface analysis curves $\left(\mathrm{N}_{2}\right.$ adsorption, $77 \mathrm{~K}$ ) of birnessite and MnTAPP@bir. The specific surface area of the birnessite is $35.623 \mathrm{~m}^{2} \mathrm{~g}^{-1}$. The specific surface of reassembled birnessite is $72.856 \mathrm{~m}^{2} \mathrm{~g}^{-1}$. Visibly, when birnessite exfoliated into a single layer, and then with MnTAPP self-assembly, the specific surface is more than doubled.

\subsection{Possible formation mechanism of MnTAPP@bir}

Based on the above characterization and analysis results, we speculated the formation mechanism of MnTAPP@bir 

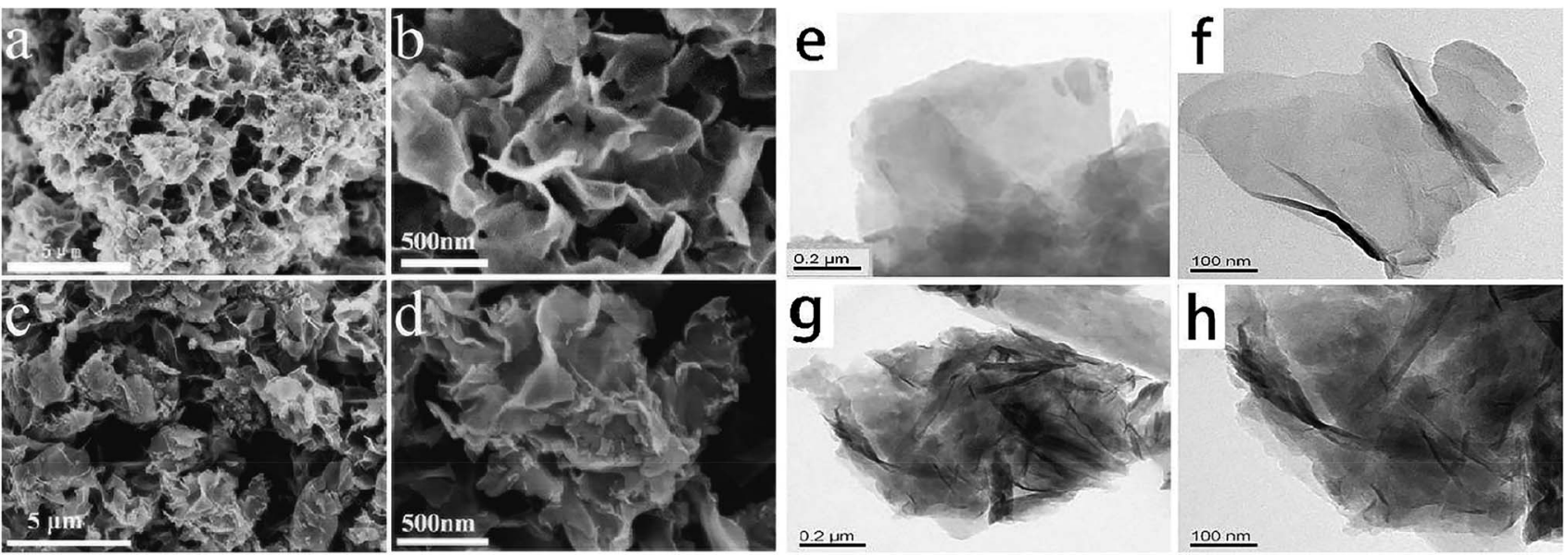

Fig. 3 SEM images. (a) and (b) are SEM photographs of birnessite; (c) and (d) are SEM photographs of MnTAPP(abir; (e) and ( $f$ ) are TEM photographs of exfoliated birnessite; $(\mathrm{g})$ and $(\mathrm{h})$ are TEM photographs of MnTAPP@bir.

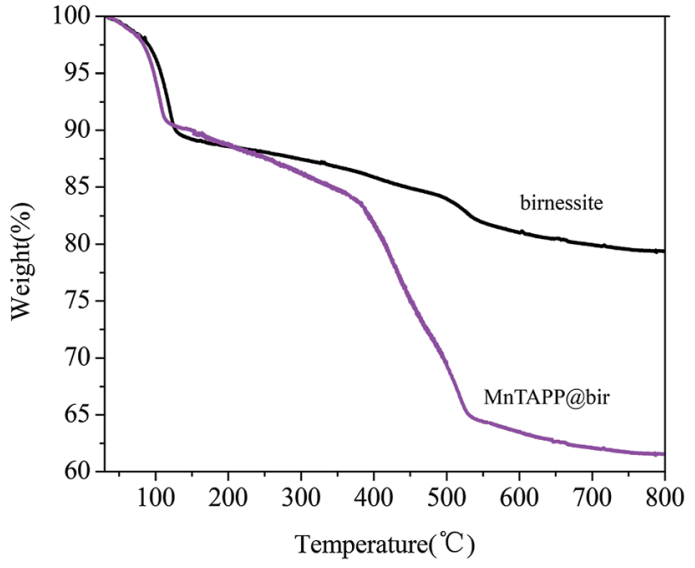

Fig. 4 TGA curves of MnTAPP@ibir and birnessite.

composites, as shown in Fig. 6. The interlayers of birnessite contain $\mathrm{Na}^{+}$and water molecules. After acidification, $\mathrm{Na}^{+}$is exchanged with $\mathrm{H}^{+}$. When tetramethylammonium (TMA) hydroxide is added, $\mathrm{TMA}^{+}$was inserted into the interlayer due to electrostatic force, which caused a rapid expansion between layers. Under the effect of water flushing, the electrostatic attraction between the layers is insufficient to maintain the layered structure, resulting in the layered structure being exfoliated. The exfoliated sheet is stably presented in the solution. The exfoliated birnessite nanoflakes have a negative charge, MnTAPP has a positive charge, and the planar MnTAPP molecules are rich in $\mathrm{NH}_{2}$ groups and have a strong coordination affinity for the Mn ions on the layer. Driven by the electrostatic force and the coordination effect of amino nitrogen on the manganese ion in birnessite, the new nanocomposite MnTAPP@bir is automatically reassembled as sandwich-type structure.

\subsection{OER application of MnTAPP@bir conductive composites}

Electrochemical measurements were carried out in a threeelectrode system on an electrochemical workstation in $1.0 \mathrm{M}$ $\mathrm{KOH}$ aqueous solution at room temperature. Glassy carbon (GC) electrodes were used as working electrodes. All the
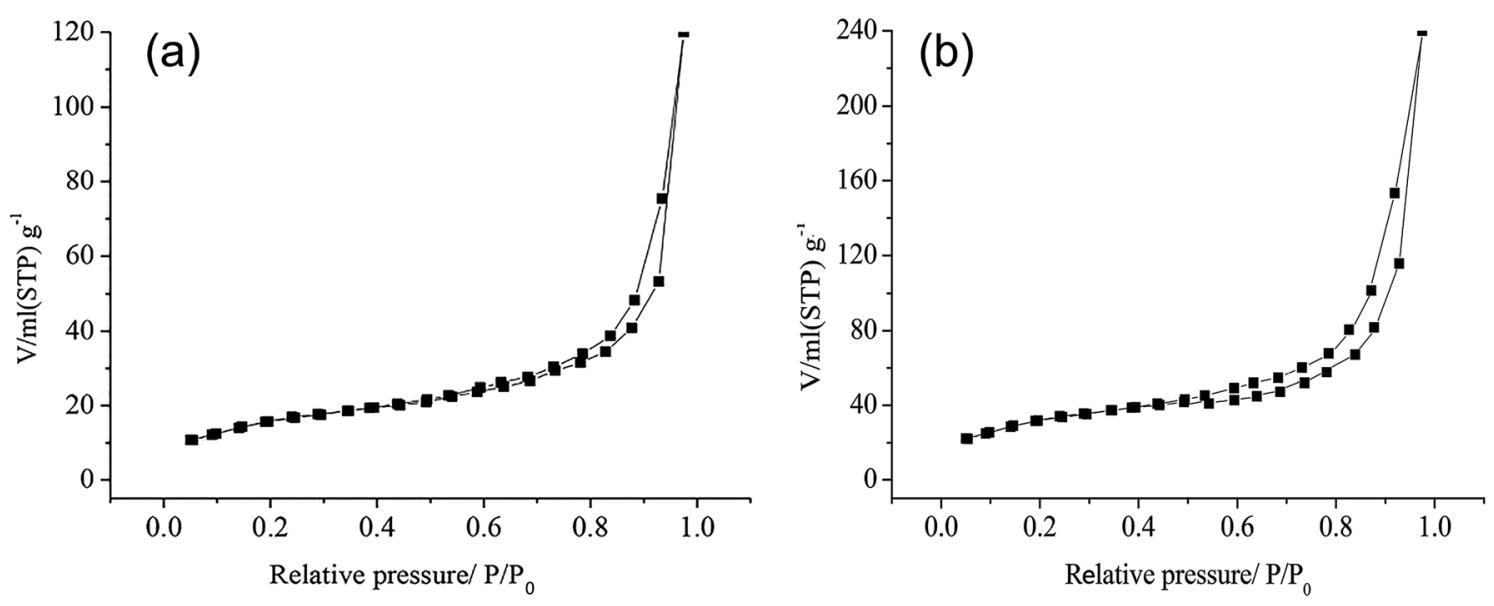

Fig. 5 BET images. BET patterns of (a) birnessite and (b) MnTAPP@ibir. 


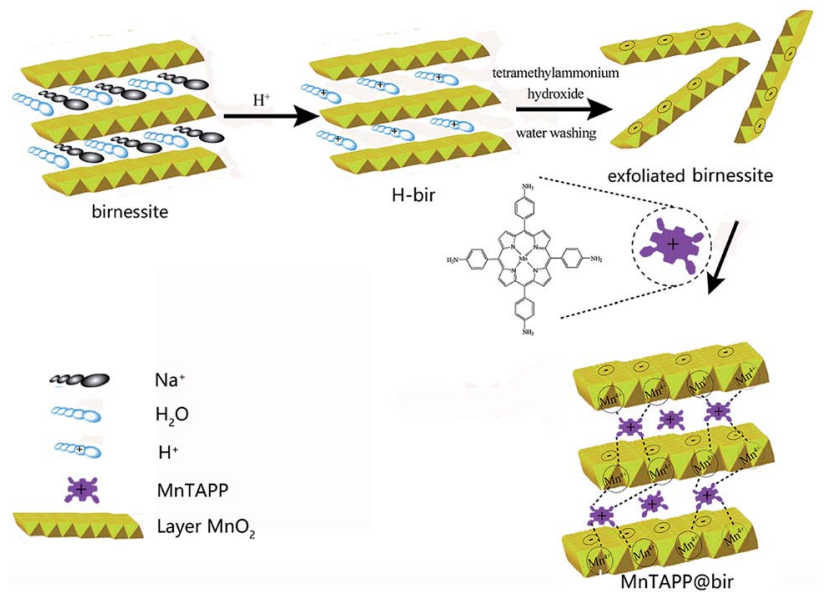

Fig. 6 Formation progress of MnTAPP@ibir.

potentials were measured with respect to a standard calomel reference electrode and Pt wire served as the counter electrode. The electrochemical behaviors of the prepared electrodes were characterized by cyclic voltammetry (CV), linear-sweep voltammetry (LSV), electrochemical impedance spectroscopy (EIS) and chronoamperometry. CV curves were measured in a potential range from 0 to $0.6 \mathrm{~V}$ at the scan rate of $5 \mathrm{mV} \mathrm{s}^{-1}$, linear-sweep voltammetry (LSV) were performed by sweeping the potential from 1.2 to $1.9 \mathrm{~V}$ at a sweep rate of $5 \mathrm{mV} \mathrm{s}^{-1}$ and an electrode rotating speed of $1600 \mathrm{rpm}$. EIS measurements were carried out in a frequency range from $0.01 \mathrm{~Hz}$ to $100 \mathrm{kHz}$ by applying an $\mathrm{AC}$ voltage with an amplitude of $5 \mathrm{mV}^{-1}$ at $0.60 \mathrm{~V}$ (vs. SCE). The chronoamperometry were conducted at $0.65 \mathrm{~V}$ ( $v s$. SCE) under the current density of about $10 \mathrm{~mA} \mathrm{~cm}{ }^{-2}$. All the polarization curves were recorded at $5 \mathrm{mV} \mathrm{s}^{-1}$ scan rate. For all the catalysts tested here, polarization curves were replicated 5 times.

From Fig. 7, it can be clearly seen that the peak area of MnTAPP@bir is significantly greater than that of birnessite (about twice its value), indicating that MnTAPP@bir has more redox active sites. The main reason for this result is that with the excellent electron transfer properties of MnTAPP, the charge transfer performance of the composite birnessite is significantly

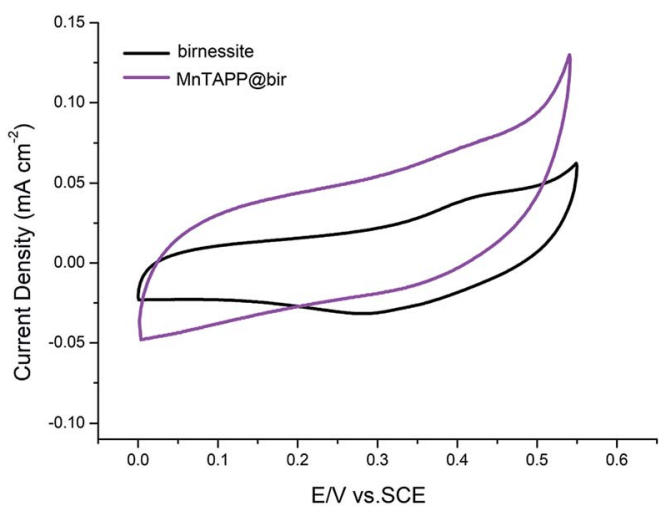

Fig. 7 Cyclic voltammograms of birnessite and MnTAPP@bir at a sweep rate of $5 \mathrm{mV} \mathrm{s}^{-1}$. enhanced, and the crystal structure of the material is changed, resulting in a significant synergistic catalytic effect. Additionally, MnTAPP provided a smooth electronic pathway for reactants and products, thereby decreasing ohmic loss and mass loss. ${ }^{38}$

In the LSV curve, the MnTAPP@bir exhibited an overpotential for water oxidation of $450 \mathrm{mV}$ (at $10 \mathrm{~mA} \mathrm{~cm}{ }^{-2}$ ) and a Tafel slope of $121.5 \mathrm{mV} \mathrm{dec}{ }^{-1}$ compared to birnessite with $700 \mathrm{mV}$ (at $10 \mathrm{~mA} \mathrm{~cm}^{-2}$ ) and $230.0 \mathrm{mV} \mathrm{dec}^{-1}$, indicating that MnTAPP@bir has the better OER property (Fig. 8). On the one hand, the birnessite has a large specific surface area after MnTAPP is compounded in the birnessite layer, it exposes and increases more catalytic active sites. On the other hand, the result of EIS (Fig. 9) also further confirmed that MnTAPP@bir has the better OER property. The observed diameter of the semi-circle of MnTAPP@bir $(\sim 360 \Omega)$ is smaller than that of birnessite $(\sim 680 \Omega)$, suggesting a much faster electron transfer during the electrochemical reaction. The conductivity is greatly enhanced so that electrocatalytic activity is greatly improved. ${ }^{39}$
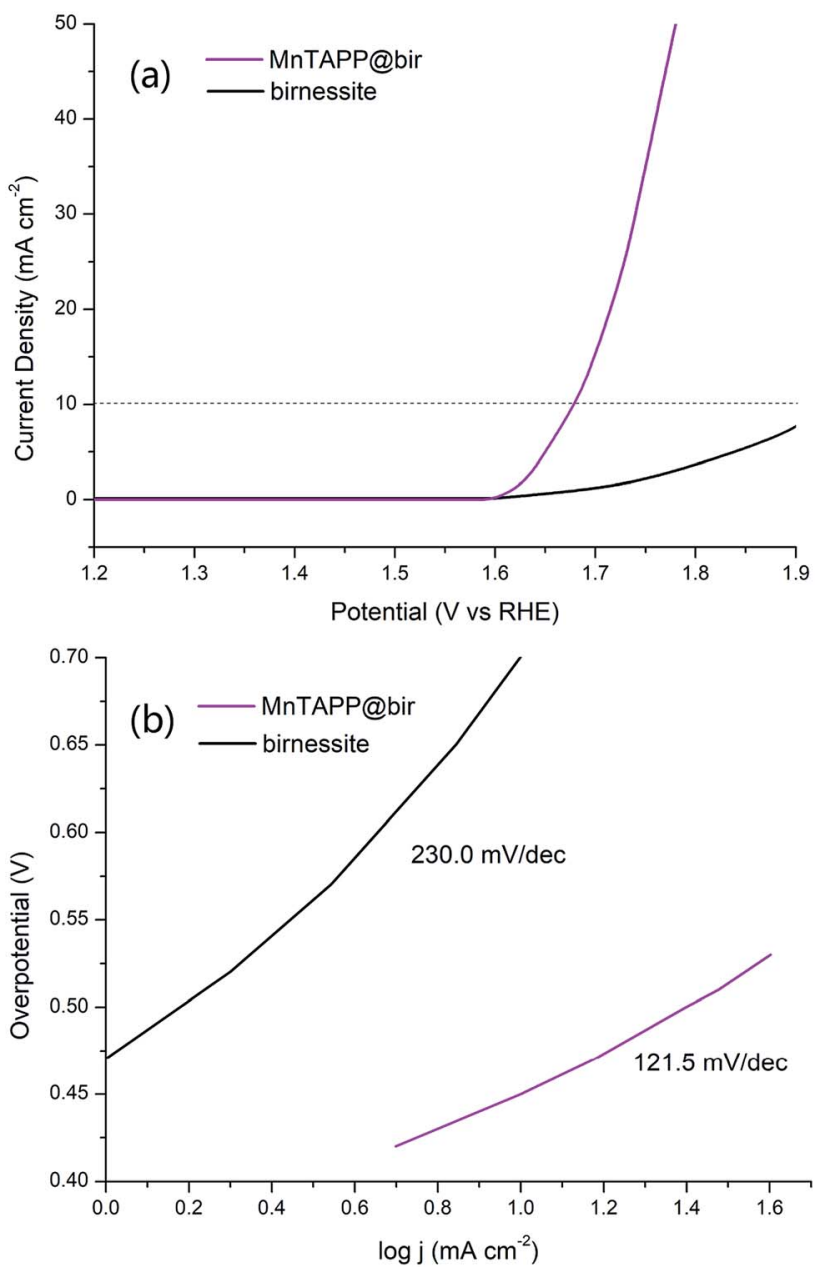

Fig. 8 (a) LSV curves of MnTAPPabir and birnessite in $1 \mathrm{M} \mathrm{KOH}$ at a sweep rate of $5 \mathrm{mV} \mathrm{s}^{-1}$; (b) Tafel plot of MnTAPP@abir and birnessite in $1 \mathrm{M} \mathrm{KOH}$ solution at a sweep rate of $5 \mathrm{mV} \mathrm{s}^{-1}$. 


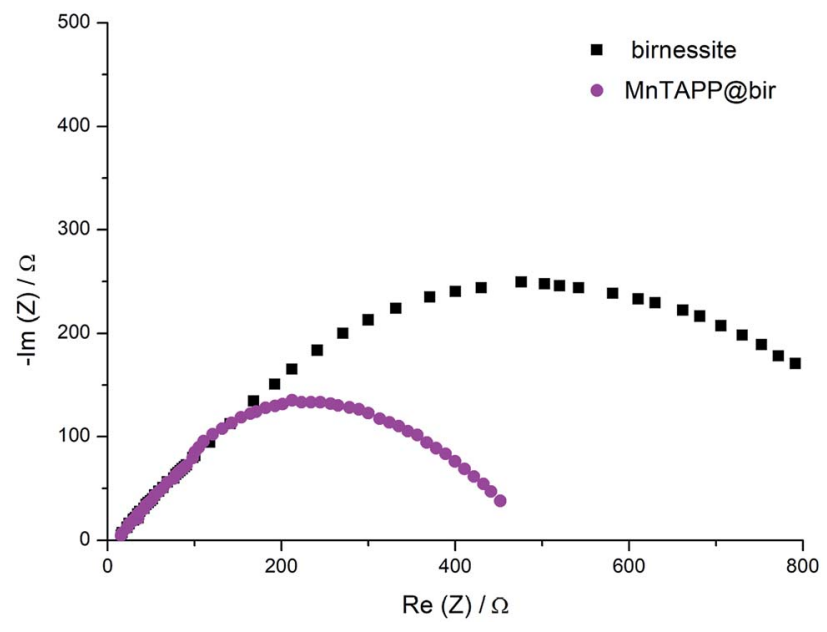

Fig. 9 Nyquist plot obtained from EIS measurement at an applied potential of $0.60 \mathrm{~V}$ (vs. SCE) for birnessite and MnTAPP@bir.

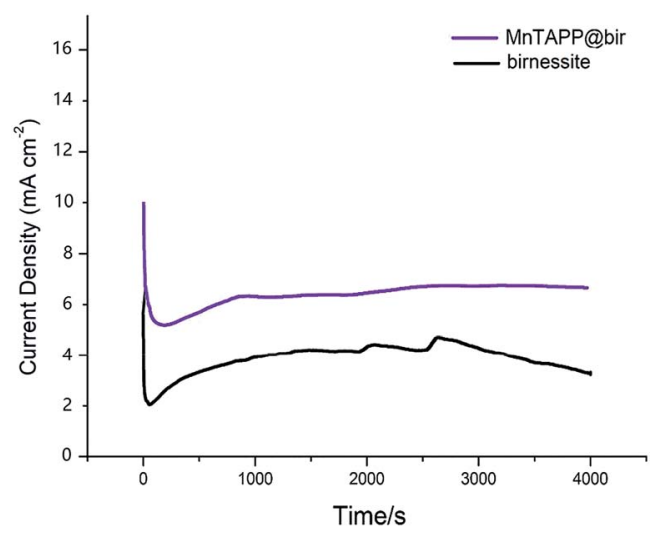

Fig. 10 Chronoamperometric curves of birnessite and MnTAPPabir at $0.65 \mathrm{~V}$ (vs. SCE) under the current density of about $10 \mathrm{~mA} \mathrm{~cm}{ }^{-2}$.

From Fig. 10, it can be seen that MnTAPP@bir exhibits better stability than birnessite in $4000 \mathrm{~s}$. When the external voltage instantaneously added to the catalyst at the initial stage, it can result in concentration polarization and ohmic polarization of the catalysts, which leads to fluctuations in the catalyst current, so the current is unstable at the initial stage. ${ }^{40}$ As the reaction proceeds, the current gradually stabilizes. In a word,

Table 1 Comparison for different non-precious metal catalysts in 1.0 $\mathrm{M} \mathrm{KOH}$ for OER

\begin{tabular}{lllll}
\hline Electrocatalyst & Electrolytes & $\begin{array}{l}\eta_{10} \\
(\mathrm{mV})\end{array}$ & $\begin{array}{l}\text { Tafel slope } \\
{\left[\mathrm{mV} \mathrm{dec}^{-1}\right]}\end{array}$ & References \\
\hline Birnessite & $1 \mathrm{M} \mathrm{KOH}$ & 700 & 230.0 & This work \\
MnTAPP@bir & $1 \mathrm{M} \mathrm{KOH}$ & 450 & 121.5 & This work \\
Zn-CNTCPP & $1 \mathrm{M} \mathrm{KOH}$ & 480 & 87.5 & 18 \\
$\mathrm{Cu}-\mathrm{CNTCPP}$ & $1 \mathrm{M} \mathrm{KOH}$ & 430 & 83.9 & 18 \\
$\mathrm{Co}^{2+}(12.2 \%) /$ birnessite & $1 \mathrm{M} \mathrm{KOH}$ & 360 & $46 \pm 3$ & 19 \\
$\mathrm{Ni}^{+2}(7.7 \%) /$ birnessite & $1 \mathrm{M} \mathrm{KOH}$ & 400 & $60 \pm 3$ & 20
\end{tabular}

MnTAPP@bir is a good OER catalyst compared to others as depicted in Table 1.

\section{Conclusions}

The high purity birnessite was exfoliated into a negatively charged monolayer structure by using tetramethylammonium hydroxide. A planar MnTAPP with positively charged and abundant $-\mathrm{NH}_{2}$ groups was synthesized. With electrostatic attraction and the coordination force of $-\mathrm{NH}_{2}$ groups on manganese ions, the self-assembled MnTAPP@bir inorganicorganic complex was successfully obtained. The structure and chemical properties of the composites were characterized by XRD, FTIR, SEM, TEM, TGA, and BET. From the analysis of the specific surface area, it can be seen that the specific surface area of self-assembled composite is greatly improved. CV, LSV, EIS and chronoamperometric measurements were performed on the MnTAPP@bir composite to investigate the catalytic water oxidation activity. The test results indicated that the new selfassembled material exhibits superior OER performance and excellent stability.

When MnTAPP was inserted into the birnessite layer, birnessite has a larger specific surface area. This aspect exposes and adds more catalytically active sites. At the same time, due to the addition of MnTAPP, the material has better conductivity, stability and catalytic activity, thus making its electrocatalytic water oxidation activity greatly improved.

\section{Conflicts of interest}

There are no conflicts to declare.

\section{Acknowledgements}

The authors would like to give thanks for the financial support from the National Natural Science Foundation of China (No. 21576074). The authors are also grateful for the financial support of Hunan University.

\section{References}

1 W. Li, Z. Cao, L. Cai, L. Zhang, X. Zhu and W. Yang, Energy Environ. Sci., 2017, 10, 101.

2 T. M. Suzuki, S. Shu, K. Sekizawa, K. Kitazumi, N. Takahashi and T. Morikawa, Appl. Catal., B, 2017, 202, 597.

3 Z. He, J. Fu, B. Cheng, J. Yu and S. Cao, Appl. Catal., B, 2017, 205, 104.

4 Y. Lu, X. Cheng, G. Tian, H. Zhao, L. He, J. Hu, S.-M. Wu, Y. Dong, G.-G. Chang and S. Lenaerts, Nano Energy, 2018, 47, 8.

5 J. Suntivich, K. J. May, H. A. Gasteiger, J. B. Goodenough and Y. Shao-Horn, Science, 2011, 334, 1383.

6 Y. Lee, J. Suntivich, K. J. May, E. E. Perry and Y. Shao-Horn, J. Phys. Chem. Lett., 2012, 3, 399.

7 K. W. Nam, S. Kim, S. Lee, M. Salama, I. Shterenberg, Y. Gofer, J.-S. Kim, E. Yang, C. S. Park and J.-S. Kim, Nano Lett., 2015, 15, 4071. 
8 H. Boumaiza, R. Coustel, C. Despas, C. Ruby and L. Bergaoui, J. Solid State Chem., 2018, 258, 543.

9 A. R. Cavazos, M. Taillefert, Y. Tang and J. B. Glass, Mar. Chem., 2018, 202, 49.

10 A. C. Thenuwara, E. B. Cerkez, S. L. Shumlas, N. H. Attanayake, I. G. McKendry, L. Frazer, E. Borguet, Q. Kang, R. C. Remsing and M. L. Klein, Angew. Chem., Int. Ed., 2016, 55, 10381.

11 C. E. Frey, M. Wiechen and P. Kurz, Dalton Trans., 2014, 43, 4370.

12 X. Xiong, Y. Ji, M. Xie, C. You, L. Yang, Z. Liu, A. M. Asiri and X. Sun, Electrochem. Commun., 2018, 86, 161.

13 G. k. Elmaci, C. E. Frey, P. Kurz and B. l. Zümreoğlu-Karan, Inorg. Chem., 2015, 54, 2734.

14 V. S. da Silva, W. C. dos Santos Vieira, A. M. Meireles, G. M. Ucoski, S. Nakagaki, Y. M. Idemori and G. DeFreitasSilva, New J. Chem., 2017, 41, 997.

15 J. C. Kemmegne-Mbouguen, H. E. Toma, K. Araki, V. R. L. Constantino, E. Ngameni and L. Angnes, Microchim. Acta, 2016, 183, 3243.

16 K. Liu, H. Zhang, R. Xing, Q. Zou and X. Yan, ACS Nano, 2017, 11, 12840.

17 L. Cui, Q. Lin, C. S. Jin, W. Jiang, H. Huang, L. Ding, N. Muhanna, J. C. Irish, F. Wang and J. Chen, ACS Nano, 2015, 9, 4484.

18 Z. Huang, M. Zhang, H. Lin, S. Ding, B. Dong, D. Liu, H. Wang, F. Dai and D. Sun, RSC Adv., 2018, 8, 40054.

19 A. C. Thenuwara, S. L. Shumlas, N. H. Attanayake, Y. V. Aulin, I. G. McKendry, Q. Qiao, Y. Zhu, E. Borguet, M. J. Zdilla and D. R. Strongin, ACS Catal., 2016, 6, 7739.

20 A. C. Thenuwara, E. B. Cerkez, S. L. Shumlas, N. H. Attanayake, I. G. McKendry, L. Frazer, E. Borguet, Q. Kang, R. C. Remsing, M. L. Klein, M. J. Zdilla and D. R. Strongin, Angew. Chem., 2016, 55, 10381.

21 A. C. Thenuwara, S. L. Shumlas, N. H. Attanayake, E. B. Cerkez, I. G. McKendry, L. Frazer, E. Borguet, Q. Kang, M. J. Zdilla, J. Sun and D. R. Strongin, Langmuir, 2015, 31, 12807.

22 S. Kosasang, N. Ma, P. Wuamprakhon, N. Phattharasupakun, T. Maihom, J. Limtrakul and M. Sawangphruk, Chem. Commun., 2018, 54, 8575.
23 X. Yang, X. Chen, X. Zhang, W. Yang and D. G. Evans, Sens. Actuators, B, 2008, 129, 784.

24 S. Yu, M. Xi, X. Jin, K. Han, Z. Wang and H. Zhu, Catal. Commun., 2010, 11, 1125.

25 J. Ducke, A. Riss, A. Pérez Paz, K. Seufert, M. Schwarz, M. Garnica, A. Rubio and W. Auwärter, ACS Nano, 2018, 12, 2677.

26 K. A. de Freitas Castro, F. Wypych, A. Antonangelo, K. M. Mantovani, A. Bail, G. M. Ucoski, K. J. Ciuffi, T. E. Cintra and S. Nakagaki, J. Colloid Interface Sci., 2016, 478, 374.

27 B. A. Manning, S. E. Fendorf, B. Bostick and D. L. Suarez, Environ. Sci. Technol., 2002, 36, 976.

28 M. Wang, L. Zhang, W. Huang, T. Xiu, C. Zhuang and J. Shi, Chem. Eng. J., 2017, 320, 667.

29 S.-M. Chen, J.-L. Song and R. Thangamuthu, J. Electroanal. Chem., 2007, 606, 63.

30 E. Eren, J. Hazard. Mater., 2008, 159, 235.

31 A. Dias, R. G. Sá, M. C. Spitale, M. Athayde and V. S. Ciminelli, Mater. Res. Bull., 2008, 43, 1528.

32 P. Di Leo, M. D. R. Pizzigallo, V. Ancona, F. Di Benedetto, E. Mesto, E. Schingaro and G. Ventruti, J. Hazard. Mater., 2012, 201, 148.

33 C. S. Diercks, S. Lin, N. Kornienko, E. A. Kapustin, E. M. Nichols, C. Zhu, Y. Zhao, C. J. Chang and O. M. Yaghi, J. Am. Chem. Soc., 2018, 140, 1116.

34 Z. Tan, J. Zhu and W. Yang, Catal. Commun., 2017, 94, 60. 35 J. Zhu, Z. Tan and W. Yang, Macromol. Res., 2017, 25, 792.

36 A. Napola, M. D. Pizzigallo, P. Di Leo, M. Spagnuolo and P. Ruggiero, Chemosphere, 2006, 65, 1583.

37 J. Wang, G. Zhang, L. Ren, L. Kang, Z. Hao, Z. Lei and Z.-H. Liu, Cryst. Growth Des., 2014, 14, 5626.

38 Z. Xi, D. P. Erdosy, A. Mendoza-Garcia, P. N. Duchesne, J. Li, M. Muzzio, Q. Li, P. Zhang and S. Sun, Nano Lett., 2017, 17, 2727.

39 Y. Meng, W. Song, H. Huang, Z. Ren, S.-Y. Chen and S. L. Suib, J. Am. Chem. Soc., 2014, 136, 11452.

40 A. Bergmann, I. Zaharieva, H. Dau and P. Strasser, Energy Environ. Sci., 2013, 6, 2745. 